\title{
Mental health and substance misuse 7 years following an Emergency Department admission for alcohol intoxication
}

\author{
A Adam", M Faouzi ${ }^{1}$, B Yersin', P Bodenmann², JB Daeppen', N Bertholet ${ }^{1}$ \\ From INEBRIA 12th Congress, \\ Atlanda, GA, USA. 24-25 September 2015
}

\section{Background}

How young adults evolve at a distance of being admitted for alcohol intoxication in the Emergency Department (ED) is not well characterized.

\section{Objective}

Assess the prevalence of alcohol use disorder (AUD), substance use and health status 7 years following an ED admission for alcohol intoxication.

\section{Methods}

In 2006-2007, 631 patients aged 18-30 were admitted for alcohol intoxication at the ED of a tertiary Swiss hospital. In 2014, they were re-contacted and interviewed to complete: demographics, alcohol use disorders identification test-consumption (AUDIT-C), Mini International Neuropsychiatric interview (MINI) for AUD, SF12 mental and physical component summary scores (MCS, PCS), Patient Health Questionnaire (depression and anxiety disorders), past year use of illegal drugs/tobacco, if they remembered the admission and discussing their drinking while admitted.

\section{Results}

In $2014,318 / 631(50.4 \%)$ patients completed the questionnaire: $32.1 \%$ were women, $36.5 \%$ unemployed, $73.6 \%$ remembered the admission and $34.6 \%$ discussing their drinking; $65.1 \%$ had AUDIT-C $\geq 4$ (i.e. positive screen for AUD). According to the MINI, $15.1 \%$ had alcohol dependence and $13.2 \%$ harmful use. $18.6 \%$ had depression, and $15.4 \%$ an anxiety disorder. Mean (SD) PCS and MCS were 52.2(9.3) and 42.7(11.7). Prevalence of any use (past year) was $80.2 \%$ for tobacco, $53.1 \%$ for cannabis, $22.6 \%$ for cocaine, $13.5 \%$ for sedatives, $11.0 \%$ for stimulants, $7.2 \%$ for opioids, and $6.0 \%$ for hallucinogens. At least once a week use was $65.4 \%$ for tobacco, $25.5 \%$ for cannabis, $3.8 \%$ for cocaine, $7.2 \%$ for sedatives, $0.9 \%$ for stimulants $3.8 \%$ for opioids and $0 \%$ for hallucinogens. No differences were found between those who completed the questionnaire and those who did not on 2006-2007 alcohol intoxication admission data (age, gender, blood alcohol concentration, presence of disruptive behavior in the ED).

\section{Conclusions}

Young patients admitted for alcohol intoxication are likely to develop substance misuse, mental health disorders, and social problems, suggesting they should be offered secondary prevention measures.

\section{Authors' details}

${ }^{1}$ Lausanne University Hospital, Lausanne, Switzerland. ${ }^{2}$ Department of Community Medicine, University of Lausanne, Lausanne, Switzerland.

Published: 24 September 2015

\section{doi:10.1186/1940-0640-10-S2-P3}

Cite this article as: Adam et al:: Mental health and substance misuse 7 years following an Emergency Department admission for alcohol intoxication. Addiction Science \& Clinical Practice 2015 10(Suppl 2):P3. 